\title{
Single- $\Lambda$ Hypernuclei in the Relativistic Mean-Field Theory with parameter set FSU
}

\author{
Renli $\mathbf{X u}^{1}$, Chen $\mathbf{W u}^{2}$, Zhongzhou Ren ${ }^{1,3,4}$ \\ ${ }^{1}$ Department of Physics, Nanjing University, Nanjing 210093, China \\ ${ }^{2}$ Shanghai Institute of Applied Physics, Chinese Academy of Sciences, Shanghai \\ 201800, China \\ ${ }^{3}$ Center of Theoretical Nuclear Physics, National Laboratory of Heavy-Ion \\ Accelerator, Lanzhou 730000, China \\ ${ }^{4}$ Kavli Institute for Theoretical Physics China, Beijing 100190, China \\ E-mail: wuchenoffd@gmail.com
}

\begin{abstract}
.
In this work, we conduct a study on the properties of single- $\Lambda$ hypernuclei in the relativistic mean-field theory with the parameter set FSU, where the isoscalar-isovector coupling has been included to soften the symmetry energy. In our model, a tensor coupling between $\omega$ and $\Lambda$ is employed, which is found to be essential in obtaining the small spin-orbit interaction in single- $\Lambda$ hypernuclei. Our calculated values of $\Lambda$ single-particle energies are in good agreement with the known experimental data. As a comparison to other existing parameter sets, calculations are also carried out by using parameterizaions such as NL3 and NL3*. The results show that the parameter set FSU is as successful as those of NL3 and NL3* in terms of reproducing the properties of single- $\Lambda$ hypernuclei.
\end{abstract}

PACS numbers: 23.60.+e, 21.10.Tg, 21.60.Gx

Submitted to: J. Phys. G: Nucl. Part. Phys. 


\section{Introduction}

The Relativistic Mean-Field Theory (RMF) has been widely used to study the properties of finite nuclei and nuclear matter and it has achieved great success [1, 2, 3, 4]. With several parameters, the RMF theory gives good description of the properties of nuclei, such as binding energy, rms radii, and single-particle energy levels. It can also be used to investigate the properties of $\Lambda$ hypernuclei as well as multi-lambda hypernuclei[5]. Since there exist only a few experimental data for hyperon-nucleon (YN) and hyperonhyperon (YY) interactions, the study of hypernuclei provides important information on the YN and YY interactions. In fact, exploring the nuclear systems with strangeness has long attracted lots of attentions and theoretical progresses have been made over the past years [5, 6, 7, 8, 9, 10]. On the experimental side, the recent observation of an antimatter hypernucleus ${ }_{\Lambda}^{3} \overline{\mathrm{H}}$ in relativistic heavy-ion collisions by STAR Collaboration[11] has aroused much interest. Besides its astrophysical and cosmological implications, strange nuclear physics is indeed an attractive topic by itself.

We know that baryons interact via the exchange of mesons in the RMF framework. In $\Lambda$ hypernuclei, the baryons include nucleons and $\Lambda$ hyperons. For the nucleonic sector, we will make use of the parameter set FSU, which was proposed by Todd-Rutel and Piekarewicz in 2005. The parameter set NL3[12, 13] and recently the improved version NL3* 14] are considered to be very successful for finite nuclei. For comparison purpose, calculations will also be carried out using these parameterizations. The FSU parameter set predicts a compression modulus for symmetric nuclear matter of $K=230$ $\mathrm{MeV}$ and a neutron skin in ${ }^{208} \mathrm{~Pb}$ with a thickness of $R_{n}-R_{p}=0.21 \mathrm{fm}$ [15], while the thickness of neutron skin in ${ }^{208} \mathrm{~Pb}$ was estimated to be $0.28 \mathrm{fm}$ by the NL3[12, 16]. The additional isoscalar-isovector coupling $\left(\Lambda_{\mathrm{v}}\right)$ term in FSU is introduced to soften the symmetry energy at high densities [15]. The validity of parameter set FSU has been examined by calculating the properties of nuclear matter, finite nuclei in some previous works [17, 18, and our aim in this work is to use the parameter set FSU as well as the NL3 and the NL3* to study the properties of single- $\Lambda$ hypernuclei.

It is widely believed that the spin-orbit splitting in $\Lambda$ hypernuclei is quite small. The tensor coupling between $\omega$ and $\Lambda$ acts as a spin-orbit interaction, and has an important impact on the spin-orbit splitting of hyperons, as observed by Jennings [19]. Following this line of thought, in our calculation we evaluate the effective potenials of hyperon in hypernuclei, particularly the spin-orbit potentials, in order to study the effect of the tensor coupling on the spin-orbit splitting of $\Lambda$ hyperon in hypernuclei.

The organization of this paper is as follows. In Sec. 2, we outline the theoretical framework of $\Lambda$ hypernuclei in RMF theory, where tensor coupling is introduced in addition to the FSU parameter set. The model parameters and numerical results are presented and discussed in Sec. 3. A summary is given in Sec. 4. 
Single- $\Lambda$ Hypernuclei in the Relativistic Mean-Field Theory

\section{Formulas of the RMF model with hyperon}

In the RMF theory, the nuclear interaction is usually described by the exchange of three mesons: the isoscalar meson $\sigma$, the isoscalar-vector meson $\omega$, and the isovector-vector meson $\rho$. There exists electromagnetic interaction between protons in nuclei, so the photon $A$ is included. The baryons involved in the present work are nucleons and $\Lambda$ hyperon. The $\Lambda$ hyperon is a charge neutral and isoscalar particle so that it does not couple to isovector-vector $\rho$ and photon A. The effective Lagrangian density is in the following form:

$$
\begin{aligned}
\mathcal{L}=\bar{\psi} & {\left[\gamma^{\mu}\left(i \partial_{\mu}-g_{\omega} \omega_{\mu}-\frac{g_{\rho}}{2} \tau \cdot \rho_{\mu}-\frac{e}{2}\left(1+\tau_{3}\right) A_{\mu}\right)-M_{N}^{*}\right] \psi } \\
& +\bar{\psi}_{\Lambda}\left[\gamma^{\mu}\left(i \partial_{\mu}-g_{\omega}^{\Lambda} \omega_{\mu}\right)-M_{\Lambda}^{*}+\frac{f_{\omega}^{\Lambda}}{2 M_{\Lambda}} \sigma^{\mu \nu} \partial_{\nu} \omega_{\mu}\right] \psi_{\Lambda} \\
& +\frac{1}{2} \partial^{\mu} \sigma \partial_{\mu} \sigma-\frac{1}{2} m_{\sigma}^{2} \sigma^{2}-\frac{1}{4} V^{\mu \nu} V_{\mu \nu}+\frac{1}{2} m_{\omega}^{2} \omega^{\mu} \omega_{\mu} \\
& -\frac{1}{4} b^{\mu \nu} b_{\mu \nu}+\frac{1}{2} m_{\rho}^{2} \rho^{\mu} \rho_{\mu}-\frac{1}{4} F^{\mu \nu} F_{\mu \nu}-U_{e f f}\left(\sigma, \omega^{\mu}, \rho^{\mu}\right)
\end{aligned}
$$

where the effective mass for the $\Lambda$ hyperon and nucleon are defined as: $M_{N}^{*}=M_{N}-g_{\sigma} \sigma$, $M_{\Lambda}^{*}=M_{\Lambda}-g_{\sigma}^{\Lambda} \sigma$. The strength tensors of the vector mesons and electromagnetic field are defined as: $V_{\mu \nu}=\partial_{\mu} \omega_{\nu}-\partial_{\nu} \omega_{\mu}, b_{\mu \nu}=\partial_{\mu} \rho_{\nu}-\partial_{\nu} \rho_{\mu}, F_{\mu \nu}=\partial_{\mu} A_{\nu}-\partial_{\nu} A_{\mu}$. The selfinteracting terms of $\sigma, \omega$ mesons and the isoscalar-isovector intertaction one are taken as:

$$
\begin{aligned}
U_{\text {eff }}\left(\sigma, \omega^{\mu}, \rho^{\mu}\right)= & \frac{\kappa}{3 !}\left(g_{\sigma} \sigma\right)^{3}+\frac{\lambda}{4 !}\left(g_{\sigma} \sigma\right)^{4}-\frac{\zeta}{4 !}\left(g_{\omega}^{2} \omega_{\mu} \omega^{\mu}\right)^{2} \\
& -\Lambda_{\mathrm{v}}\left(g_{\rho}^{2} \rho_{\mu} \rho^{\mu}\right)\left(g_{\omega}^{2} \omega_{\mu} \omega^{\mu}\right) .
\end{aligned}
$$

Here, the isoscalar meson self-interactions (via $\kappa, \lambda$, and $\zeta$ ) are necessary for the appropriate equation of state of symmetric nuclear matter [12, 20]. The new additional isoscalar-isovector coupling $\left(\Lambda_{\mathrm{v}}\right)$ term is used to modify the density dependence of the symmetry energy and the neutron skin thicknesses of heavy nuclei [15, 17]. The $\psi$ and $\psi_{\Lambda}$ are the Dirac spinors for nucleons and $\Lambda$ hyperon.

Using the mean-field approximation, the Dirac equations for nucleons and $\Lambda$ hyperon have the following form:

$$
\begin{aligned}
& {\left[i \gamma^{\mu} \partial_{\mu}-\left(M_{N}-g_{\sigma} \sigma\right)-g_{\omega} \gamma^{0} \omega_{0}-\frac{g_{\rho}}{2} \gamma^{0} \tau_{3} \rho_{0}-\frac{e}{2} \gamma^{0}\left(1+\tau_{3}\right) A_{0}\right] \psi=0,} \\
& {\left[i \gamma^{\mu} \partial_{\mu}-\left(M_{\Lambda}-g_{\sigma}^{\Lambda} \sigma\right)-g_{\omega}^{\Lambda} \gamma^{0} \omega_{0}+\frac{f_{\omega}^{\Lambda}}{2 M_{\Lambda}} \sigma^{0 i} \partial_{i} \omega\right] \psi_{\Lambda}=0}
\end{aligned}
$$

The Klein-Gordon equations for the mesons and photon can be written as

$$
\begin{aligned}
\left(-\Delta+m_{\sigma}^{2}\right) \sigma(\mathbf{r}) & =g_{\sigma} \rho_{s}(\mathbf{r})+g_{\sigma}^{\Lambda} \rho_{s}^{\Lambda}(\mathbf{r})-\frac{\kappa}{2} g_{\sigma}^{3} \sigma^{2}(\mathbf{r})-\frac{\lambda}{6} g_{\sigma}^{4} \sigma^{3}(\mathbf{r}), \\
\left(-\Delta+m_{\omega}^{2}\right) \omega_{0}(\mathbf{r}) & =g_{\omega} \rho_{v}(\mathbf{r})+g_{\omega}^{\Lambda} \rho_{v}^{\Lambda}(\mathbf{r})-\frac{\zeta}{6} g_{\omega}^{4} \omega_{0}^{3}(\mathbf{r}) \\
& -2 \Lambda_{\mathrm{v}} g_{\rho}^{2} g_{\omega}^{2} \rho_{0}^{2}(\mathbf{r}) \omega_{0}(\mathbf{r})+\frac{f_{\omega}^{\Lambda}}{2 M_{\Lambda}} \rho_{0}^{T}(\mathbf{r}),
\end{aligned}
$$


Single- $\Lambda$ Hypernuclei in the Relativistic Mean-Field Theory

$$
\begin{aligned}
& \left(-\Delta+m_{\rho}^{2}\right) \rho_{0}(\mathbf{r})=\frac{g_{\rho}}{2} \rho_{3}(\mathbf{r})-2 \Lambda_{\mathrm{v}} g_{\rho}^{2} g_{\omega}^{2} \omega_{0}^{2}(\mathbf{r}) \rho_{0}(\mathbf{r}), \\
& -\Delta A_{0}(\mathbf{r})=e \rho_{p}(\mathbf{r}) .
\end{aligned}
$$

where $\rho_{s}(\mathbf{r})\left(\rho_{s}^{\Lambda}(\mathbf{r})\right), \rho_{v}(\mathbf{r})\left(\rho_{v}^{\Lambda}(\mathbf{r})\right), \rho_{0}^{T}(\mathbf{r}), \rho_{3}(\mathbf{r})$ and $\rho_{p}(\mathbf{r})$ are the scalar, vector, tensor, third component of isovector, and proton densities, respectively. Eqs.(3) and (4) can be self-consistently solved by iteration. We focus our study on the spherical case, and use the BCS theory to calculate the pairing contribution for open shell nuclei. The detail of the solution can be easily found in the literatures [1, 21], and it is not reiterated here.

Table 1: The parameter sets NL3, NL3*, and FSU. The nucleon and rho masses are respectively set as $M_{N}=939 \mathrm{MeV}, m_{\rho}=763$

$\mathrm{MeV}$.

\begin{tabular}{lccccccccc}
\hline & $m_{\sigma}(\mathrm{MeV})$ & $m_{\omega}(\mathrm{MeV})$ & $g_{\sigma}^{2}$ & $g_{\omega}^{2}$ & $g_{\rho}^{2}$ & $\kappa(\mathrm{MeV})$ & $\lambda$ & $\zeta$ & $\Lambda_{\mathrm{v}}$ \\
\hline NL3 & 508.1940 & 782.501 & 104.3871 & 165.5854 & 80.0667 & 3.8599 & -0.0159 & 0.000 & 0.000 \\
NL3 $^{*}$ & 502.5742 & 782.600 & 101.8969 & 164.0064 & 83.7152 & 4.1474 & -0.0174 & 0.000 & 0.000 \\
FSU & 491.5000 & 782.500 & 112.1996 & 204.5469 & 138.4701 & 1.4203 & 0.0238 & 0.060 & 0.030 \\
\hline
\end{tabular}

\section{Numerical results and discussion}

In our calculation, we use the parameter sets NL3, NL3* and FSU [15, 22] for the parameters of the nucleonic sector, whose are tabulated in table 1. At first, we define the ratios of the meson-hyperon couplings to the meson-nucleon couplings, $R_{\sigma}=g_{\sigma}^{\Lambda} / g_{\sigma}$ and $R_{\omega}=g_{\omega}^{\Lambda} / g_{\omega}$. We take the naive quark model value for the relative $\omega$ coupling as $R_{\omega}=2 / 3$ [23, 24]. To reproduce the experimental $\Lambda$ binding energies of single- $\Lambda$ hypernuclei, we adopt the relative $\sigma$ coupling as $R_{\sigma}=0.619$ for the FSU, $R_{\sigma}=0.620$ for the NL3* and $R_{\sigma}=0.621$ for the NL3 parameter set. We take the experimental nucleon mass as $M_{N}=939 \mathrm{MeV}$ and lambda hyperon mass as $M_{\Lambda}=1116 \mathrm{MeV}$, respectively.

Table 2: $\Lambda$ single-particle energies(in $\mathrm{MeV}$ ) for nuclear core plus one $\Lambda$ configuration. The values for ${ }_{\Lambda}^{16} \mathrm{O},{ }_{\Lambda}^{40} \mathrm{Ca},{ }_{\Lambda}^{48} \mathrm{Ca},{ }_{\Lambda}^{51} \mathrm{~V},{ }_{\Lambda}^{89} \mathrm{Y}$, ${ }_{\Lambda}^{139} \mathrm{La}$ and ${ }_{\Lambda}^{208} \mathrm{~Pb}$ are calculated. The available experimental data denoted by a are taken from Ref. [25] and denoted by b are taken from Ref.[26] .

\begin{tabular}{cccccc}
\hline & $\Lambda$ state & NL3 & NL3 ${ }^{*}$ & FSU & Expt. \\
\hline${ }^{16} \mathrm{O}$ & $1 s_{1 / 2}$ & -12.91 & -12.64 & -12.88 & $-12.5 \pm 0.35^{a}$ \\
& $1 p_{3 / 2}$ & -2.96 & -2.88 & -2.49 & \\
& $1 p_{1 / 2}$ & -2.73 & -2.66 & -2.26 & $-2.5 \pm 0.5^{a}$ \\
\hline
\end{tabular}

(Continued on next page) 
Single- $\Lambda$ Hypernuclei in the Relativistic Mean-Field Theory

Table 2 - continued from previous page

\begin{tabular}{|c|c|c|c|c|c|}
\hline & $\Lambda$ state & NL3 & NL3 $^{*}$ & FSU & Expt. \\
\hline \multirow[t]{6}{*}{${ }^{4{ }^{40} \mathrm{Ca}}$} & $\overline{1 s_{1 / 2}}$ & $\begin{array}{c}-19.44 \\
\end{array}$ & -19.28 & $\begin{array}{c}-19.33 \\
\end{array}$ & $-18.7 \pm 1.1^{a}$ \\
\hline & $1 p_{3 / 2}$ & -10.59 & -10.39 & -10.26 & \\
\hline & $1 p_{1 / 2}$ & -10.34 & -10.14 & -10.04 & $-11.0 \pm 0.6^{a}$ \\
\hline & $1 d_{5 / 2}$ & -2.62 & -2.52 & -2.07 & \\
\hline & $2 s_{1 / 2}$ & -2.40 & -2.41 & -2.10 & \\
\hline & $1 d_{3 / 2}$ & -2.35 & -2.26 & -1.81 & $-1.0 \pm 0.5^{a}$ \\
\hline \multirow[t]{6}{*}{${ }_{\Lambda}^{48} \mathrm{Ca}$} & $1 s_{1 / 2}$ & -21.08 & -20.88 & -20.87 & \\
\hline & $1 p_{3 / 2}$ & -12.53 & -12.31 & -12.22 & \\
\hline & $1 p_{1 / 2}$ & -12.31 & -12.09 & -12.03 & \\
\hline & $1 d_{5 / 2}$ & -4.37 & -4.23 & -3.85 & \\
\hline & $2 s_{1 / 2}$ & -3.64 & -3.63 & -3.21 & \\
\hline & $1 d_{3 / 2}$ & -4.08 & -3.95 & -3.57 & \\
\hline \multirow[t]{6}{*}{${ }_{\Lambda}^{51} \mathrm{~V}$} & $1 s_{1 / 2}$ & -21.82 & -21.64 & -21.39 & $-19.9 \pm 1.0^{a}$ \\
\hline & $1 p_{3 / 2}$ & -13.29 & -13.06 & -12.87 & \\
\hline & $1 p_{1 / 2}$ & -13.08 & -12.85 & -12.69 & \\
\hline & $1 d_{5 / 2}$ & -4.98 & -4.83 & -4.43 & \\
\hline & $2 s_{1 / 2}$ & -4.06 & -4.05 & -3.56 & \\
\hline & $1 d_{3 / 2}$ & -4.67 & -4.53 & -4.14 & $-4.0 \pm 0.5^{a}$ \\
\hline \multirow[t]{7}{*}{${ }_{\Lambda}^{89} \mathrm{Y}$} & $1 s_{1 / 2}$ & -24.05 & -23.90 & -23.66 & $-23.1 \pm 0.5^{b}$ \\
\hline & $1 p_{3 / 2}$ & -17.47 & -17.25 & -17.13 & \\
\hline & $1 p_{1 / 2}$ & -17.34 & -17.11 & -17.03 & $-16.5 \pm 4.1^{b}$ \\
\hline & $1 d_{5 / 2}$ & -10.47 & -10.26 & -10.02 & \\
\hline & $2 s_{1 / 2}$ & -8.82 & -8.73 & -8.21 & \\
\hline & $1 d_{3 / 2}$ & -10.23 & -10.02 & -9.80 & $-9.1 \pm 1.3^{b}$ \\
\hline & $1 f_{5 / 2}$ & -3.22 & -3.08 & -2.66 & $-2.3 \pm 1.2^{b}$ \\
\hline \multirow[t]{9}{*}{${ }_{\Lambda}^{139} \mathrm{La}$} & $1 s_{1 / 2}$ & -25.29 & -25.09 & -24.94 & $-24.5 \pm 1.2^{b}$ \\
\hline & $1 p_{3 / 2}$ & -20.37 & -20.14 & -20.06 & \\
\hline & $1 p_{1 / 2}$ & -20.32 & -20.09 & -20.01 & $-20.4 \pm 0.6^{b}$ \\
\hline & $1 d_{5 / 2}$ & -14.65 & -14.41 & -14.29 & \\
\hline & $2 s_{1 / 2}$ & -12.51 & -12.34 & -12.04 & \\
\hline & $1 d_{3 / 2}$ & -14.51 & -14.27 & -14.17 & $-14.3 \pm 0.6^{b}$ \\
\hline & $1 f_{7 / 2}$ & -8.50 & -8.29 & -8.04 & \\
\hline & $1 f_{5 / 2}$ & -8.26 & -8.05 & -7.82 & $-8.0 \pm 0.6^{b}$ \\
\hline & $1 g_{7 / 2}$ & -1.98 & -1.84 & -1.44 & $-1.5 \pm 0.6^{b}$ \\
\hline \multirow[t]{9}{*}{${ }_{\Lambda}^{208} \mathrm{~Pb}$} & $1 s_{1 / 2}$ & -26.69 & -26.52 & -26.26 & $-26.3 \pm 0.8^{b}$ \\
\hline & $1 p_{3 / 2}$ & -22.67 & -22.46 & -22.28 & \\
\hline & $1 p_{1 / 2}$ & -22.63 & -22.41 & -22.24 & $-21.9 \pm 0.6^{b}$ \\
\hline & $1 d_{5 / 2}$ & -17.89 & -17.65 & -17.51 & \\
\hline & $2 s_{1 / 2}$ & -15.91 & -15.73 & -15.51 & \\
\hline & $1 d_{3 / 2}$ & -17.79 & -17.55 & -17.42 & $-16.8 \pm 0.7^{b}$ \\
\hline & $1 f_{7 / 2}$ & -12.60 & -12.36 & -12.17 & \\
\hline & $1 f_{5 / 2}$ & -12.42 & -12.18 & -12.01 & $-11.7 \pm 0.6^{b}$ \\
\hline & $1 g_{7 / 2}$ & -6.78 & -6.58 & -6.28 & $-6.6 \pm 0.6^{b}$ \\
\hline
\end{tabular}

In the present calculation, we adopt the quark model value of the tensor coupling, $f_{\omega}^{\Lambda}=-g_{\omega}^{\Lambda}$ [19, 27]. The center-of-mass correction to the binding energy is given as 
$E_{c . m .}=-\frac{3}{4} \times 41 A^{-1 / 3} \mathrm{MeV}$.

The $\Lambda$ binding energy is a very important quantity for the investigation of the properties of $\Lambda$ hypernuclei. We first calculate the $\Lambda$ single-particle energies for some typical spherical hypernuclei. The results are shown in table 2 . By putting the $\Lambda$ particle either in the $1 s_{1 / 2}$ orbit or in one of the other orbits, we can get the corresponding $\Lambda$ single-particle energy. One can easily see from table 2 that the $\Lambda$ binding energies in our calculation are in good agreement with the experimental value and the parameter set FSU is as good as those of NL3 and NL3* for the binding energies of the $\Lambda$ hypernuclei. We also find that when the nuclear number increases, the $\Lambda$ binding energy in $\Lambda$ hypernuclei for $1 s_{1 / 2}$ state becomes smaller, and finally approaches the binding energy for $\Lambda$ bounded in nuclear matter with $\epsilon_{\Lambda}=-28 \mathrm{MeV}$ [18, 28.

The small spin-orbit splittings of $\Lambda$ hyperon are also obtained in our calculation. As usually undertood, the spin-orbit splitting of the p orbit is quite small, $-0.8 \mathrm{MeV}$ $\leq \delta_{\Lambda} \leq 0.2 \mathrm{MeV}$ [26], where $\delta_{\Lambda}=\epsilon_{\Lambda}\left(p_{1 / 2}\right)-\epsilon_{\Lambda}\left(p_{3 / 2}\right)$. In Ref. [26], the energy difference between the $2_{1}^{+}$and $0_{1}^{+}$states of ${ }_{\Lambda}^{16} \mathrm{O}$ is obtained to be $0.04 \pm 0.32 \mathrm{MeV}$. From table 2, we can see that the $\delta_{\Lambda}$ of $1 \mathrm{p}$ orbit for ${ }_{\Lambda}^{16} \mathrm{O}$ is about $-0.23 \mathrm{MeV}$ for NL3, NL3* and FSU, which is consistent with the results in Ref. [26]. As a comparison, we also calculate the $\Lambda$ binding energies without tensor coupling between $\omega$ and $\Lambda$, where the result of $\delta_{\Lambda}$ of $1 \mathrm{p}$ orbit for ${ }_{\Lambda}^{16} \mathrm{O}$ is $-1.41 \mathrm{MeV}$ with the FSU, which is much bigger than case with tensor coupling. Therefore we can conclude that the tensor coupling plays an important role in the spin-orbit interaction in single- $\Lambda$ hypernuclei.

Another interesting result concerns the $\Lambda$ binding energies of $2 s_{1 / 2}$ and $1 d_{3 / 2}$. The $\Lambda$ binding energy of $2 s_{1 / 2}$ is bigger than $1 d_{3 / 2}$ for ${ }_{\Lambda}^{40} \mathrm{Ca}$, while it is smaller in the case of ${ }_{\Lambda}^{48} \mathrm{Ca},{ }_{\Lambda}^{51} \mathrm{~V},{ }_{\Lambda}^{89} \mathrm{Y},{ }_{\Lambda}^{139} \mathrm{La}$ and ${ }_{\Lambda}^{208} \mathrm{~Pb}$. What makes it important is that the results calculated in all of the three parameter sets ( NL3, NL3* FSU) show the same phenomena. This can be understood as a result of the presence of tensor coupling. As one can see from table 2 that the spin-orbit splitting of $1 \mathrm{~d}$ level of $\Lambda$ hyperon reduces as the nuclear number increases. As a consequence, the energy level of the $\Lambda$ orbit $1 d_{3 / 2}$ is pulled down, and the difference of energy levels between $2 s_{1 / 2}$ and $1 d_{3 / 2}$ switches its sign.

We know that the spin-orbit interaction of baryons contain contributions from the tensor potential and the derivative of the sum of the scalar and vector potentials. By recasting the Dirac equation in Schrödinger equivalent form, we can obtain the spin-orbit potential of $\Lambda$ hyperon $V_{l s}^{\Lambda} l \cdot s$, with [29].

$$
\begin{aligned}
& V_{l s}^{\Lambda} l \cdot s=\frac{1}{2 M_{e f f}^{2}}\left[\frac{1}{r}\left(g_{\omega}^{\Lambda} \frac{\partial \omega_{0}}{\partial r}+g_{\sigma}^{\Lambda} \frac{\partial \sigma}{\partial r}+2 f_{\omega \Lambda} \frac{M_{e f f}}{M_{\Lambda}} \frac{\partial \omega_{0}}{\partial r}\right)\right] l \cdot s, \\
& M_{e f f}=M_{\Lambda}-\frac{1}{2}\left(g_{\omega}^{\Lambda} \omega_{0}+g_{\sigma}^{\Lambda} \sigma\right) .
\end{aligned}
$$

The scalar and vector potentials of the $\Lambda$ hyperon for ${ }_{\Lambda}^{16} \mathrm{O},{ }_{\Lambda}^{40} \mathrm{Ca}$ and ${ }_{\Lambda}^{208} \mathrm{~Pb}$ are plotted in Fig.1, where the solid curves are for scalar potentials and the dotted ones for vector potentials, the $1 p_{3 / 2} \Lambda$ state are also shown for comparison. From Fig.1, we can see that the difference between the scalar and vector potentials near the center of the hypernuclei is typically $\sim 30-35 \mathrm{MeV}$, which is consistent with the result calculated 

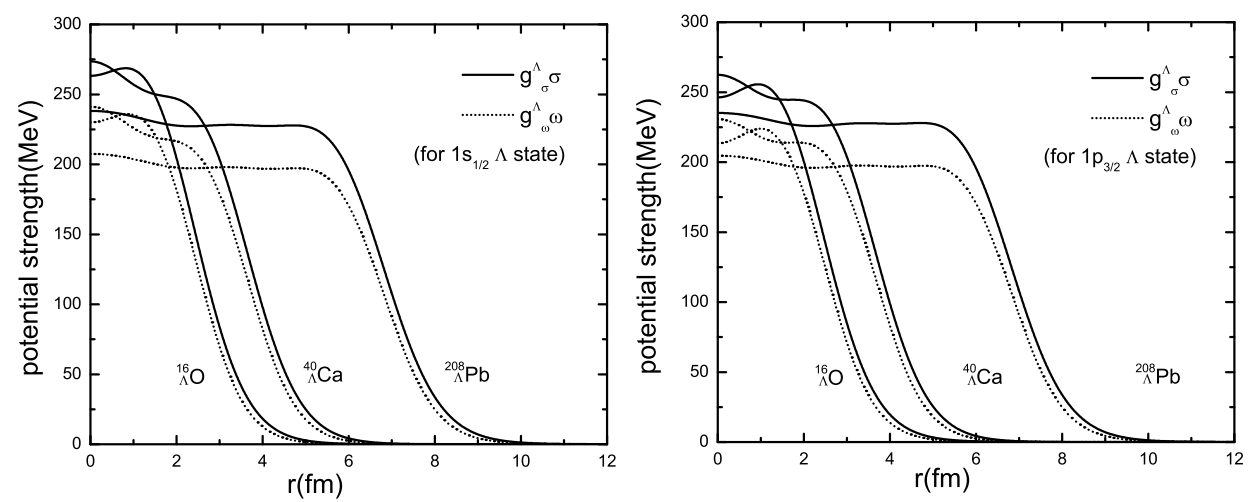

Figure 1. Scalar and vector potentials of $\Lambda$ in ${ }_{\Lambda}^{16} \mathrm{O}$ and ${ }_{\Lambda}^{40} \mathrm{Ca}$ and ${ }_{\Lambda}^{208} \mathrm{~Pb}$, respectively. The results of $1 p_{3 / 2} \Lambda$ state are also shown for comparison. The using parameter set is FSU.
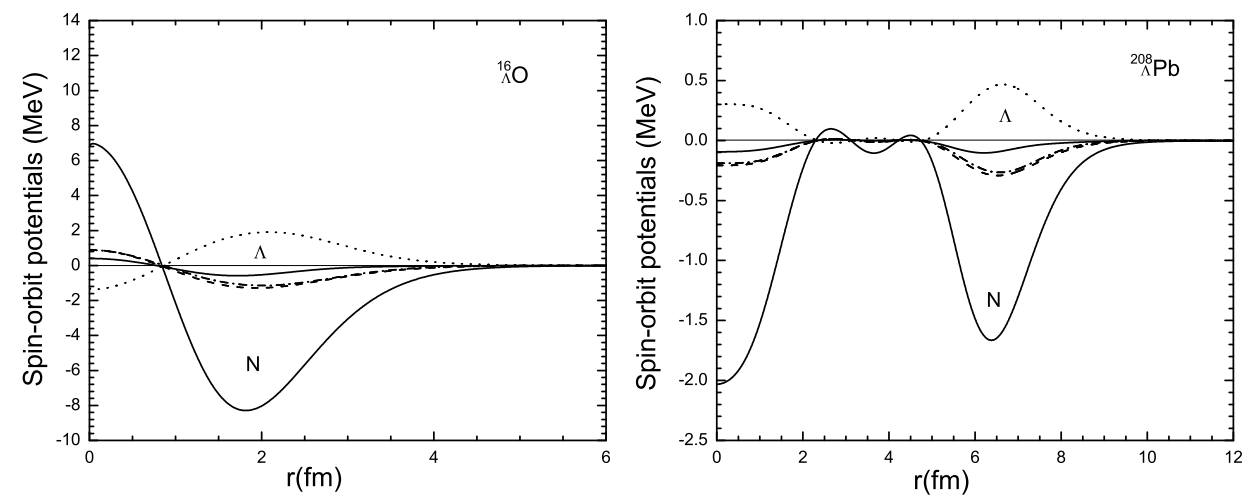

Figure 2. Spin-orbit potentials of baryons in ${ }_{\Lambda}^{16} \mathrm{O}$ and ${ }_{\Lambda}^{208} \mathrm{~Pb}$. The solid curves are the total spin-orbit potentials for a proton and a $\Lambda$. The dotted, dash-dotted and dashed ones are those for a $\Lambda$ produced by the tensor, vector and scalar potentials, respectively. All cases are for the $1 s_{1 / 2} \Lambda$ state. The using parameter set is FSU.

by Ma et al. [30]. Additionally, the scalar and vector potentials near the center of the hypernuclei in $1 p_{3 / 2} \Lambda$ state is smaller than those in $1 s_{1 / 2} \Lambda$ state, which cause the root-mean-square (rms) radius of the $\Lambda\left(r_{\Lambda}\right)$ in $1 p_{3 / 2}$ state become bigger than in $1 s_{1 / 2}$ state.

The spin-orbit potentials for ${ }_{\Lambda}^{16} \mathrm{O}$ and ${ }_{\Lambda}^{208} \mathrm{~Pb}$ are shown in Fig.2, where dashdotted, dashed, dotted curves are produced by first, second and third terms in Eq.(5), respectively. From Fig.2, we can see that the tensor potentials is comparable in magnitude with scalar and vector potentials, however, it has a negative sign. As a result, the spin-orbit term for $\Lambda$ became very small. It is also seen from Fig. 2 that the spin-orbit splitting of proton in ${ }_{\Lambda}^{16} \mathrm{O}$ is much bigger than that in ${ }_{\Lambda}^{208} \mathrm{~Pb}$. For example, 
$\delta_{P}\left(\delta_{P}=\epsilon_{P}\left(p_{1 / 2}\right)-\epsilon_{P}\left(p_{3 / 2}\right)\right)$ of 1 p state for ${ }_{\Lambda}^{16} \mathrm{O}$ is $-6.99 \mathrm{MeV}$, but it is only $-0.61 \mathrm{MeV}$ for ${ }_{\Lambda}^{208} \mathrm{~Pb}$ in our calculation.

Table 3: Binding energy per baryon, $\mathrm{E} / \mathrm{A}($ in $\mathrm{MeV})$, rms charge radius $\left(r_{c}\right)$, and rms radii of the $\Lambda\left(r_{\Lambda}\right)$, neutron $\left(r_{n}\right)$, proton $\left(r_{p}\right)$ (in $\mathrm{fm})$. The results are calculated in FSU.

\begin{tabular}{ccccccc}
\hline & $\Lambda$ state & $E / A$ & $r_{c}$ & $r_{\Lambda}$ & $r_{n}$ & $r_{p}$ \\
\hline${ }_{\Lambda}^{17} \mathrm{O}$ & $1 s_{1 / 2}$ & -8.25 & 2.68 & 2.42 & 2.53 & 2.56 \\
${ }_{\Lambda}^{17} \mathrm{O}$ & $1 p_{3 / 2}$ & -7.65 & 2.69 & 3.69 & 2.54 & 2.57 \\
${ }_{16}^{16} \mathrm{O}$ & & -7.96 & 2.69 & & 2.54 & 2.56 \\
\hline${ }_{\Lambda}^{41} \mathrm{Ca}$ & $1 s_{1 / 2}$ & -8.78 & 3.43 & 2.66 & 3.28 & 3.33 \\
${ }_{\Lambda}^{41} \mathrm{Ca}$ & $1 p_{3 / 2}$ & -8.56 & 3.44 & 3.49 & 3.29 & 3.34 \\
${ }_{40} \mathrm{Ca}$ & & -8.52 & 3.43 & & 3.29 & 3.34 \\
\hline${ }_{\Lambda}^{49} \mathrm{Ca}$ & $1 s_{1 / 2}$ & -8.82 & 3.46 & 2.74 & 3.57 & 3.37 \\
${ }_{\Lambda}^{49} \mathrm{Ca}$ & $1 p_{3 / 2}$ & -8.64 & 3.47 & 3.49 & 3.57 & 3.37 \\
${ }^{48} \mathrm{Ca}$ & & -8.57 & 3.47 & & 3.57 & 3.37 \\
\hline${ }^{90} \mathrm{Y}$ & $1 s_{1 / 2}$ & -8.85 & 4.24 & 3.16 & 4.27 & 4.16 \\
${ }_{\Lambda}^{90} \mathrm{Y}$ & $1 p_{3 / 2}$ & -8.77 & 4.24 & 3.85 & 4.27 & 4.16 \\
${ }_{\Lambda}^{89} \mathrm{Y}$ & & -8.68 & 4.24 & & 4.27 & 4.16 \\
\hline${ }_{\Lambda}^{140} \mathrm{La}$ & $1 s_{1 / 2}$ & -8.53 & 4.86 & 3.63 & 4.96 & 4.79 \\
${ }_{\Lambda}^{140} \mathrm{La}$ & $1 p_{3 / 2}$ & -8.49 & 4.86 & 4.29 & 4.96 & 4.79 \\
${ }_{139} \mathrm{La}$ & & -8.41 & 4.87 & & 4.97 & 4.80 \\
\hline${ }_{\Lambda}^{209} \mathrm{~Pb}$ & $1 s_{1 / 2}$ & -7.96 & 5.53 & 4.01 & 5.67 & 5.46 \\
${ }_{\Lambda}^{209} \mathrm{~Pb}$ & $1 p_{3 / 2}$ & -7.94 & 5.53 & 4.72 & 5.67 & 5.47 \\
${ }_{208} \mathrm{~Pb}$ & & -7.87 & 5.53 & & 5.68 & 5.47 \\
\hline
\end{tabular}

In table 3, we list the calculated binding energy per baryon $\mathrm{E} / \mathrm{A}(\mathrm{MeV})$, rms charge radius $r_{c}$, and $\mathrm{rms}$ radii of the $\Lambda$, the neutron and proton distributions $\left(r_{\Lambda}, r_{n}, r_{p}\right.$, respectively), for the $1 s_{1 / 2}$ and $1 p_{3 / 2} \Lambda$ configurations. As a comparison, we also give these quantities for normal finite nuclei.

It is seen that the $\Lambda$ hyperon has a weak influence on the rms charge radius, and rms radii of the nucleon. In addition, the rms radius of the $\Lambda$ in $1 s_{1 / 2}$ state tends to be bigger as the nuclear number increases. Regarding the effects of the $\Lambda$ on the core nucleons, we also show in Fig.3 the nucleon single particle energies for ${ }^{16} \mathrm{O},{ }_{\Lambda}^{17} \mathrm{O},{ }^{40} \mathrm{Ca}$ and ${ }_{\Lambda}^{41} \mathrm{Ca}$ for $1 s_{1 / 2} \Lambda$ state. The existence of the $\Lambda$ makes the scalar and baryon densities larger, and the scalar and vector potentials become stronger. As a result, the binding energies of nucleons in ${ }_{\Lambda}^{17} \mathrm{O},{ }_{\Lambda}^{41} \mathrm{Ca}$ are more deeper than those of ${ }^{16} \mathrm{O},{ }^{40} \mathrm{Ca}$, respectively.

Finally, we show the effective masses of the nucleon and $\Lambda$ as well as the baryon densities calculated for ${ }_{\Lambda}^{41} \mathrm{Ca},{ }_{\Lambda}^{90} \mathrm{Y},{ }_{\Lambda}^{140} \mathrm{La},{ }_{\Lambda}^{209} \mathrm{~Pb}$ for the $1 s_{1 / 2} \Lambda$ state in Fig.4. We see that the effective masses in these hypernuclei behave in a similar manner as the distance $r$ from the center of each nucleus increases [31], and with increasing nuclear number, the $\Lambda$ hyperon density near the center of the hypernuclei becomes smaller. This is mainly because that the rms radius of the $\Lambda$ in $1 s_{1 / 2}$ state becomes bigger as the nuclear number 


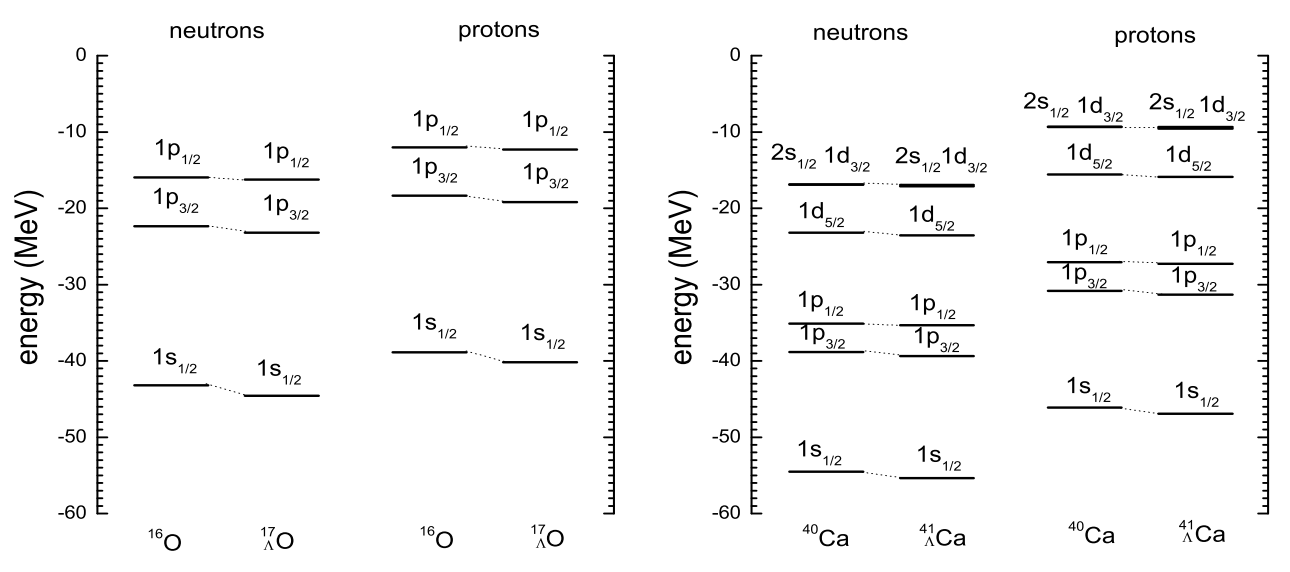

Figure 3. Nucleon single-particle energies for ${ }^{16} \mathrm{O},{ }^{40} \mathrm{Ca},{ }_{\Lambda}^{17} \mathrm{O}$ and ${ }_{\Lambda}^{41} \mathrm{Ca}$ for the $1 s_{1 / 2}$ $\Lambda$ state. The using parameter set is FSU.

increases.

\section{4. summary}

In the present work, the properties of some typical spherical hypernuclei have been systemically investigated in the RMF model with FSU parameter set as well as the NL3 and NL3* ones. We discussed the influence of the tensor coupling between $\omega$ and $\Lambda$ on the single- $\Lambda$ hypernuclei. In our calculation, we obtained that the $\delta_{\Lambda}$ of $1 \mathrm{p}$ orbit for ${ }_{\Lambda}^{16} \mathrm{O}$ is $-0.23 \mathrm{MeV}$ with the presence of tensor coupling, in contrast to the calculated value of $-1.41 \mathrm{MeV}$ when tensor coupling is switched off. From the calculation, we find that the tensor potential is comparable in magnitude with scalar and vector potentials for the $\Lambda$ hyperon in $\Lambda$ hypernuclei, but it has a negative sign, which causes the spinorbit term for $\Lambda$ to be very small. It is also shown that the tensor coupling leads to the inversion of energy levels between some states (corresponding to the pseudospin doublets) for $\Lambda$ hyperon. Our calculation also showed that the $\Lambda$ hyperon has a weak influence on the rms charge radius, and rms radii of the nucleon density distribution. The $\Lambda$ binding energies in single- $\Lambda$ hypernuclei in our calculation are in good agreement with the available experimental data. So we can see that the parameter set FSU is as successful as the NL3 and NL3* in reproducing the properties of single- $\Lambda$ hypernuclei.

\section{Acknowledgments}

We thank Prof. W. L. Qian for valuable discussion and his advice on the language of this manuscript. This work was supported by the National Natural Science Foundation of China (Grants No. 11035001, No. 10735010, No. 10975072, No. 11120101005, and No. 11105072), by the 973 National Major State Basic Research and Development 

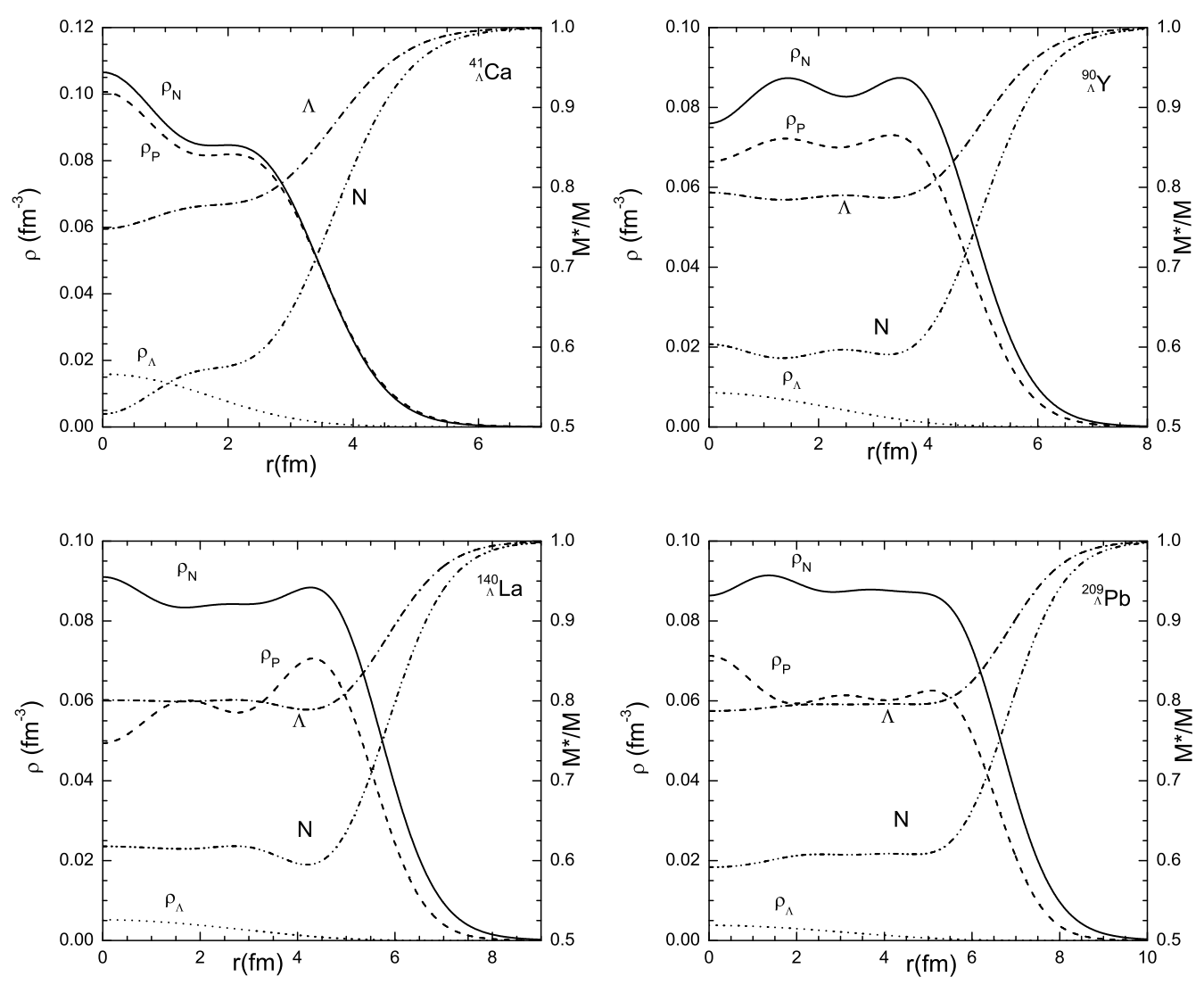

Figure 4. Calculated proton, neutron, $\Lambda$ hyperon densites, and effective masses of the nucleon denoted by, $\mathrm{N}$, and the $\Lambda$ hyperon denoted by, $\Lambda$, in hypernuclei for ${ }_{\Lambda}^{41} \mathrm{Ca}$, ${ }_{\Lambda}^{90} \mathrm{Y},{ }_{\Lambda}^{140} \mathrm{La},{ }_{\Lambda}^{209} \mathrm{~Pb}$. All cases are for the $1 s_{1 / 2} \Lambda$ state. The using parameter set is FSU.

of China (Grants No. 2007CB815004 and No. 2010CB327803), by CAS Knowledge Innovation Project No. KJCX2-SW-N02, by Research Fund of Doctoral Point, Grant No. 20100091110028, and by the Project Funded by the Priority Academic Program Development of Jiangsu Higher Education Institutions (PAPD).

\section{References}

[1] Ring P 1996 Prog. Part. Nucl. Phys. 37193.

[2] Lalazissis G A, Sharma M M, Ring P and Gambhir Y K 1996 Nucl. Phys. A 608202.

[3] Sugahara Y and Toki H 1994 Nucl. Phys. A 579557.

[4] Ren Z Z, Zhu Z Y, Cai Y H and Xu G O 1996 Phys. Lett. B 380241.

[5] Rufa M et al. 1990 Phys. Rev. C 422469.

[6] Glendenning N K, Von-Eiff D, Haft M, Lenske H and Weigel M K 1993 Phys. Rev. C 48889.

[7] Win M T, Hagino K and Koike T 2011 Phys. Rev. C 83014301.

[8] Vretenar D, Pöschl W, Lalazissis G A and Ring P 1998 Phys. Rev. C 57 R1060. 
[9] Tsushima K, Saito K, Haidenbauer J and Thomas A W 1998 Nucl. Phys. A 630691.

[10] Shen H and Toki H 2002 Nucl. Phys. A 707469.

[11] The STAR Collaboration 2010 Science 32858.

[12] Lalazissis G A, König J and Ring P 1997 Phys. Rev. C 55540.

[13] Lalazissis G A, Raman S and Ring P 1999 At. Data Nucl. Data Tables 711.

[14] Lalazissis G A et al. 2009 Phys.Lett. B 67136.

[15] Todd-Rutel B G and Piekarewicz J 2005 Phys. Rev. Lett. 95122501.

[16] Horowitz C J and Piekarewicz J 2001 Phys. Rev. Lett. 865647.

[17] Sheng Z Q, Ren Z Z and Jiang W Z 2010 Nucl. Phys. A 83249.

[18] Wu C and Ren Z Z 2011 Phys. Rev. C 83025805.

[19] Jennings B K 1990 Phys. Lett. B 246325.

[20] Toki H, Hirata D, Sugahara Y, Sumiyoshi K and Tanihata I 1995 Nucl. Phys. A 588 c357.

[21] Gambhir Y K, Ring P and Thimet A 1990 Ann. Phys. (N.Y.) 198132.

[22] Fattoyev F J, Horowitz C J, Piekarewicz J and Shen G 2010 Phys. Rev. C 82055803.

[23] Shen H, Yang F and Toki H 2006 Prog.Theor.Phys. 115325.

[24] Keil C M, Hofmann F and Lenske H 2000 Phys. Rev. C 61064309.

[25] Usmani Q N and Bodmer A R 1999 Phys. Rev. C 60055215.

[26] Hashimoto O and Tamura H 2006 Prog. Part. Nucl. Phys. 57564.

[27] Sugahara Y and Toki H Prog. Theo. Phys. 199492803.

[28] Millener D J, Dover C B and Gal A 1988 Phys. Rev. C 382700.

[29] Mareš J and Jennings B K 1994 Phys. Rev. C 492472.

[30] Ma Z Y, Speth J, Krewald S, Chen B Q and Reuber A 1996 Nucl. Phys. A 608305.

[31] Tsushima K, Saito K and Thomas A W 1997 Phys. Lett. B 4119. 